\title{
水溶液中一步合成二吡咯甲烷类化合物
}

\author{
吴振豪胡江磊王凯凯高超王洪宇张 龙* \\ (长春工业大学吉林省石化资源与生物质综合利用工程实验室 长春 130012)
}

\begin{abstract}
摘要 在水相中, 以磷铇酸为催化剂, 吡咯与羰基化合物为底物, 室温下一步合成了二吡咯甲烷类化合物, 反应产物 纯度高, 无需进一步纯化. 该方法具有明显的环境友好、产率高和操作过程简便的优点.
\end{abstract}

关键词 二吡咯甲烷; 吡咯; 羰基化合物; 水; 绿色化学

\section{One-Step Synthesis of Dipyrromethanes in Water}

\author{
Wu, Zhenhao Hu, Jianglei Wang, Kaikai Gao, Chao Wang, Hongyu Zhang, Long* \\ (Jilin Provincial Engineering Laboratory for the Complex Utilization of Petro Resources and Biomass, \\ Changchun University of Technology, Changchun 130012)
}

\begin{abstract}
A novel and non-conventional synthesis of meso-substitued dipyrromethanes under the catalysis of $\mathrm{H}_{3} \mathrm{PW}_{12} \mathrm{O}_{40}$ in water at room temperature is described in this paper. The reactions were performed under mild conditions, affording various dipyrrometnanes in good to excellent yields and high purity.

Keywords dipyrromethane; pyrrole; carbonyl compound; water; green chemistry
\end{abstract}

二吡咯甲烷类化合物是合成卟啉、corroles、吓啉的 衍生物, 比如邻联吡啶类、杯吓啉类、叶绿素类等物质 的重要前体 ${ }^{[1 \sim 6]} .1994$ 年, Lindsey 等 ${ }^{[7]}$ 首次合成了 meso取代的二吡咯甲烷类化合物, 并将其成功应用于各种对 称与不对称卟啉的合成中, 合成了一系列的新型卟啉化 合物. 随后, 又陆续出现了一些合成 meso-取代二吡咯 甲烷类化合物的新方法 ${ }^{[8 \sim 17]}$, 但绿色、简便、高效率的 合成方法还未见报道.

生物体内发生的大量、复杂、精确的生化反应通常 是以水作为介质的, 近年来有机化学家们对如何使用水 来替代现在被广泛用作有机反应介质的有机溶剂进行 了大量的研究工作. 水作为反应介质已经成功应用于四 烷基 meso-取代卟啉的合成 ${ }^{[18]}$ 、 Michacel 加成反应 ${ }^{[19,20]}$, 锌催化的化学选择性还原硝基化合物的反应 ${ }^{[21]}$ 、Raney $\mathrm{Ni}-\mathrm{Al}$ 催化还原芳环化合物的反应 ${ }^{[22]}$, 氯联苯的去氯化 反应 ${ }^{[23]}$ 等反应中, 甚至与格氏反应相类似的反应 ${ }^{[24]}$ 也 可以使用水作为反应介质.

$\mathrm{Xu}$ 及其合作者 ${ }^{[25]}$ 研究了水为反应介质, 铋为催化 剂, 苯甲醛参与的 Mannich 反应, 我们从中得到启发, 开发了一种以吡咯和羰基化合物为起始原料, 磷铇酸为 催化剂，在水相中室温高效合成 meso-取代二吡咯甲烷
类化合物的新方法(Scheme 1).

$$
\begin{aligned}
& \text { 1a 1h } \\
& \mathrm{R}^{1}=\mathrm{H}, \mathrm{R}^{2}=4-\mathrm{NO}_{2} \mathrm{C}_{6} \mathrm{H}_{4}(\mathbf{3 a}), 4-\mathrm{HOC}_{6} \mathrm{H}_{4}(\mathbf{3 b}), \mathrm{C}_{6} \mathrm{H}_{5}(\mathbf{c} \mathbf{c}) ; \\
& \mathrm{R}^{1}=\mathrm{CH}_{3}, \mathrm{R}^{2}=\mathrm{CH}_{3}(\mathbf{3 d}), \mathrm{C}_{6} \mathrm{H}_{5}(\mathbf{3 e}), 4-\mathrm{NO}_{2} \mathrm{C}_{6} \mathrm{H}_{4}(\mathbf{3 f}), \\
& 4-\mathrm{CH}_{3} \mathrm{OC}_{6} \mathrm{H}_{4}(\mathbf{3 g}), 4-\mathrm{H}_{2} \mathrm{NC}_{6} \mathrm{H}_{4}(\mathbf{3 h})
\end{aligned}
$$

\section{Scheme 1}

\section{1 实验部分}

\section{1 试剂与仪器}

所用试剂均为市售分析纯产品, 其中磷铇酸为 Aldrich 产品, 去离子水为自制. 熔点数据由上海鹏顺科 学仪器有限公司的 X-6 自动熔点测定仪测定, 经标准品 校正; ${ }^{1} \mathrm{H}$ NMR 和 ${ }^{13} \mathrm{C}$ NMR 数据由 Bruker AMX-400 $\mathrm{MHz}$ 核磁共振仪测定, TMS 为内标; 元素分析数据由美 国 PE2400 II 型元素分析仪测定.

\section{2 实验过程}

典型的实验过程如下所示: 向三口烧瓶中加入 100 $\mathrm{mL}$ 去离子水, 通氮气 $10 \mathrm{~min}$ 以去除水中的氧气; 氮气

*E-mail: zhanghongzhl@163.com

Received July 1, 2011; revised August 27, 2011; accepted November 23, 2011. 
保护下分别加入 $0.15 \mathrm{~g}(1 \mathrm{mmol})$ 对硝基苯甲醛, $0.4 \mathrm{~mL}$ (6 mmol)新蒸的吡咯和 $0.1 \mathrm{~mol}$ 的磷钨酸, 密封, 控制温 度在 $25{ }^{\circ} \mathrm{C}$ 后开始摚拌; TLC 监测到反应完成后加入适 量氨水淬灭反应, 过滤, 滤饼用蒸馏水洗涤 5 次, $40{ }^{\circ} \mathrm{C}$ 真空干燥后得到相应的二吡咯甲烷类化合物粗产品, 利 用 ${ }^{1} \mathrm{H} N M R$ 分析其纯度. 已知化合物的结构由熔点、 ${ }^{1} \mathrm{H}$ NMR 数据与文献数据进行比较后确定, 新化合物的结 构由熔点, ${ }^{1} \mathrm{H}$ NMR, ${ }^{13} \mathrm{C}$ NMR 及元素分析数据确定.

\section{3 产物的表征}

产物的表征数据如下, 其中 $\mathbf{3 a} \sim \mathbf{3} \mathbf{c}$ 为已知化合物, $\mathbf{3 d} \sim \mathbf{3 h}$ 为新化合物.

5-(4-硝基苯基)二吡咯甲烷(3a): m.p. $144 \sim 146{ }^{\circ} \mathrm{C}$ (lit. $\left.{ }^{[17]}: 144 \sim 147{ }^{\circ} \mathrm{C}\right) ;{ }^{1} \mathrm{H}$ NMR (400 MHz, $\mathrm{CDCl}_{3}$ ) $\delta: 5.58$ (s, $1 \mathrm{H}$, meso-H), 5.87 (d, $J=5.7 \mathrm{~Hz}, 2 \mathrm{H}, 2 \mathrm{C} 3-\mathrm{H}), 6.17$ (dd, $J=2.8,5.7 \mathrm{~Hz}, 2 \mathrm{H}, 2 \mathrm{C} 4-\mathrm{H}), 6.74(\mathrm{dd}, J=2.8,1.2 \mathrm{~Hz}, 2 \mathrm{H}$, 2C5-H), 7.36 (d, $J=8.6 \mathrm{~Hz}, 2 \mathrm{H}, \mathrm{HAr}$ ), 7.98 (br s, 2H, $\mathrm{NH}), 8.16$ (d, $J=8.6 \mathrm{~Hz}, 2 \mathrm{H}, \mathrm{ArH})$.

5-(4-羟基苯基)二吡咯甲烷(3b): m.p. 108 $110{ }^{\circ} \mathrm{C}$ (lit. $\left.{ }^{[17]}: 108 \sim 110{ }^{\circ} \mathrm{C}\right) ;{ }^{1} \mathrm{H}$ NMR $\left(400 \mathrm{MHz}, \mathrm{CDCl}_{3}\right) \delta: 5.25$ (s, 1H, OH), $5.34(\mathrm{~s}, 1 \mathrm{H}), 5.87(\mathrm{~s}, 2 \mathrm{H}), 6.67(\mathrm{~s}, 2 \mathrm{H}), 6.13$ (q, $J=3.0 \mathrm{~Hz}, 2 \mathrm{H}), 6.63 \sim 6.69(\mathrm{~m}, 4 \mathrm{H}), 7.01(\mathrm{~d}, J=8.5$ $\mathrm{Hz}, 2 \mathrm{H}), 7.93$ (s, 2H, NH).

5-苯基二吡咯甲烷(3c): m.p. $109 \sim 110{ }^{\circ} \mathrm{C}$ (lit. ${ }^{[17]}$ : $\left.109 \sim 111{ }^{\circ} \mathrm{C}\right) ;{ }^{1} \mathrm{H}$ NMR $\left(400 \mathrm{MHz}, \mathrm{CDCl}_{3}\right) \delta: 5.47(\mathrm{~s}, 1 \mathrm{H})$, 6.15 (q, $J=2.9 \mathrm{~Hz}, 2 \mathrm{H}), 6.69$ (t, $J=2.4 \mathrm{~Hz}, 2 \mathrm{H}), 7.20 \sim$ 7.33 (m, 5H, ArH), 7.92 (s, 2H, NH).

5,5-二甲基二吡咯甲烷(3d): m.p. $56 \sim 57{ }^{\circ} \mathrm{C} ;{ }^{1} \mathrm{H}$ NMR $\left(400 \mathrm{MHz}, \mathrm{CDCl}_{3}\right) \delta: 1.65(\mathrm{~s}, 6 \mathrm{H}), 6.10(\mathrm{~d}, J=5.7$ $\mathrm{Hz}, 2 \mathrm{H}), 6.36$ (dd, $J=2.8,5.7 \mathrm{~Hz}, 2 \mathrm{H}), 7.78$ (br s, $2 \mathrm{H}) ;{ }^{13} \mathrm{C}$ NMR $\left(\mathrm{CDCl}_{3}\right) \delta: 29.3,35.3,103.7,107.7,117.1,139.1$. Anal. calcd for $\mathrm{C}_{11} \mathrm{H}_{14} \mathrm{~N}_{2}$ : C 75.82, H 8.10, N 16.08; found C 75.63, H 8.23, N 16.14.

5-甲基-5-苯基二吡咯甲烷(3e): m.p. $110 \sim 111{ }^{\circ} \mathrm{C}$; ${ }^{1} \mathrm{H}$ NMR (400 MHz, $\left.\mathrm{CDCl}_{3}\right) \delta: 2.05\left(\mathrm{~s}, 3 \mathrm{H}, \mathrm{CH}_{3}\right), 5.97(\mathrm{~d}$, $\left.J=5.7 \mathrm{~Hz}, 2 \mathrm{H}, 2 \mathrm{C}^{3}-\mathrm{H}\right), 6.17(\mathrm{dd}, J=2.8,5.7 \mathrm{~Hz}, 2 \mathrm{H}$, $\left.2 \mathrm{C}^{4}-\mathrm{H}\right), 6.67\left(\mathrm{dd}, J=2.8,1.2 \mathrm{~Hz}, 2 \mathrm{H}, 2 \mathrm{C}^{5}-\mathrm{H}\right), 7.12(\mathrm{~d}, J=$ $8.6 \mathrm{~Hz}, 2 \mathrm{H}, \mathrm{ArH}), 7.27$ (br s, 4H, ArH), 7.77 (s, 2H, NH). ${ }^{13} \mathrm{C}$ NMR $\left(\mathrm{CDCl}_{3}\right) \delta: 28.9,44.8,106.3,108.2,116.9$, 126.7, 127.4, 128.1, 137.5, 147.3. Anal. calcd for $\mathrm{C}_{16} \mathrm{H}_{16} \mathrm{~N}_{2}$ : C 81.32, H 6.82, N 11.85; found C 81.15, H 6.93, N 11.91 .

5-甲基-5-(4-硝基苯基)二吡咯甲烷(3f)：m.p. 142 $143{ }^{\circ} \mathrm{C} ;{ }^{1} \mathrm{H}$ NMR (400 MHz, $\mathrm{CDCl}_{3}$ ) $\delta: 2.07$ (s, 3H, $\mathrm{CH}_{3}$ ), $5.94\left(\mathrm{~d}, J=5.7 \mathrm{~Hz}, 2 \mathrm{H}, 2 \mathrm{C}^{3}-\mathrm{H}\right), 6.20(\mathrm{dd}, J=2.8,5.7 \mathrm{~Hz}$, $\left.2 \mathrm{H}, 2 \mathrm{C}^{4}-\mathrm{H}\right), 6.72\left(\mathrm{dd}, J=2.8,1.2 \mathrm{~Hz}, 2 \mathrm{H}, 2 \mathrm{C}^{5}-\mathrm{H}\right), 7.28(\mathrm{~d}$,
$J=8.6 \mathrm{~Hz}, 4 \mathrm{H}, \mathrm{ArH}), 7.84$ (br s, 2H, NH), 8.12 (s, 2H, $\mathrm{ArH}) ;{ }^{13} \mathrm{C}$ NMR (400 MHz, $\mathrm{CDCl}_{3}$ ) $\delta: 28.5,45.1,107.0$, 108.6, 117.8, 123.2, 128.4, 135.8, 146.7, 155.1. Anal. calcd for $\mathrm{C}_{16} \mathrm{H}_{15} \mathrm{~N}_{3} \mathrm{O}_{2}$ : C 68.31, H 5.37, N 14.94; found C 68.41, H 5.34, N 14.87 .

5- 甲基-5-(4- 甲氧基苯基)二吡咯甲烷 (3g): m.p. $128 \sim 129{ }^{\circ} \mathrm{C} ;{ }^{1} \mathrm{H}$ NMR $\left(400 \mathrm{MHz}, \mathrm{CDCl}_{3}\right) \delta: 2.03$ (s, 3H, $\left.\mathrm{CH}_{3}\right), 3.79$ (s, 3H, $\left.\mathrm{CH}_{3}\right), 5.97\left(\mathrm{~d}, J=5.7 \mathrm{~Hz}, 2 \mathrm{H}, 2 \mathrm{C}^{3}-\mathrm{H}\right)$, 6.17 (dd, $J=2.8,5.7 \mathrm{~Hz}, 2 \mathrm{H}, 2 \mathrm{C}^{4}-\mathrm{H}$ ), 6.66 (dd, $J=2.8,1.2$ $\mathrm{Hz}, 2 \mathrm{H}, 2 \mathrm{C}^{5}-\mathrm{H}$ ), 6.81 (d, $\left.J=8.6 \mathrm{~Hz}, 4 \mathrm{H}, \mathrm{ArH}\right), 7.03$ (br s, $2 \mathrm{H}, \mathrm{ArH}), 7.77$ (s, 2H, NH); ${ }^{13} \mathrm{C}$ NMR (400 MHz, $\mathrm{CDCl}_{3}$ ) $\delta$ : 29.0, 44.1, 55.2, 106.1, 108.2, 113.4, 116.8, 128.5, 137.8, 139.4, 158.2. Anal. calcd for $\mathrm{C}_{17} \mathrm{H}_{18} \mathrm{~N}_{2} \mathrm{O}_{1}$ : C 76.66, H 6.81, N 10.52; found C 76.41, H 6.87, N 10.61 .

5-甲基-5-(4-胺基苯基)二吡咯甲烷(3h): m.p. 168 $169{ }^{\circ} \mathrm{C} ;{ }^{1} \mathrm{H}$ NMR $\left(400 \mathrm{MHz}, \mathrm{CDCl}_{3}\right) \delta: 2.00\left(\mathrm{~s}, 3 \mathrm{H}, \mathrm{CH}_{3}\right)$, $3.61\left(\mathrm{~s}, 2 \mathrm{H}, \mathrm{NH}_{2}\right), 5.97\left(\mathrm{~d}, J=5.7 \mathrm{~Hz}, 2 \mathrm{H}, 2 \mathrm{C}^{3}-\mathrm{H}\right), 6.16$ (dd, $\left.J=2.8,5.7 \mathrm{~Hz}, 2 \mathrm{H}, 2 \mathrm{C}^{4}-\mathrm{H}\right), 6.57(\mathrm{dd}, J=2.8,1.2 \mathrm{~Hz}$, $2 \mathrm{H}, 2 \mathrm{C}^{5}-\mathrm{H}$ ), 6.61 (d, J=8.6 Hz, 2H, ArH), 6.89 (br s, 2H, ArH), 7.77 (s, 2H, NH); ${ }^{13} \mathrm{C}$ NMR (400 MHz, $\left.\mathrm{CDCl}_{3}\right) \delta$ : 29.0, 44.1, 106.0, 108.2, 114.8, 116.6, 128.4, 137.3, 138.1, 145.0. Anal. calcd for $\mathrm{C}_{16} \mathrm{H}_{17} \mathrm{~N}_{3}$ : C 76.46, H 6.82, N 16.72; found C 76.34, H 6.87, N 16.79.

\section{2 结果与讨论}

醛与吡咯发生缩合, 首先可以生成二吡咯甲烷类化 合物, 进一步反应能生成三吡咯甲烷类化合物及聚合程 度更高的齐聚物 ${ }^{[13]}$. Lindsey 等 ${ }^{[7]}$ 认为, 如果控制反应进 行的程度可以使反应停留在生成二吡咯甲烷类化合物 的阶段. 他为了控制反应进行的程度, 在反应中采用大 过量(醛与吡咯的物质的量比为 $1: 20 \sim 60$ ) 的吡咯代替 其他有机溶剂作为反应溶剂, 同时及时淬灭催化剂, 使 反应停留在生成二吡咯甲烷类化合物的阶段 ${ }^{[15]}$. 过量 的吡咯极大地降低了先生成的二吡咯甲烷类化合物的 浓度, 延缓了更高聚合反应进行的速度, 及时淬灭催化 剂, 使反应产物不能继续反应生成其它更高的低聚物.

由于二吡咯甲烷类化合物的水溶性极差, 因此, 我 们以水替代过量的吡咯为反应溶剂来达到降低反应产 物浓度的目的. 由于吡咯和醛在水中有一定的溶解度, 因此在水中吡咯和醛能进行缩合反应, 随着反应的不断 进行, 生成二吡咯甲烷类化合物会不断沉淀使更高的聚 合反应不能继续进行，同时沉淀的生成推动了底物的不 断溶解，使生成二吡咯甲烷类化合物的缩合反应不断进 行下去, 所以我们获得了高纯度和高收率的目的产物二 吡咯甲烷类化合物. 
为了研究该反应的影响因素，我们以对硝基苯甲醛 与吡咯的反应为模型反应, 通过改变反应条件包括醛或 酮与吡咯的比例、催化剂浓度、底物浓度进行了一系列 的重复实验.

\section{1 醛或酮与吡咯比例}

对硝基苯甲醛与吡咯的比例对转化率和产率的影 响的实验结果列于表 1 .

表 1 对硝基苯甲醛与吡咯的比例对其缩合反应结果的影响 ${ }^{a}$

Tabel 1 Effect of ratio of $p$-nitrobenzaldehyde to pyrrole on their condensation

\begin{tabular}{cccc}
\hline Entry & $n(\mathbf{1 a}): n(\mathbf{2})$ & Conversion/\% & Yield/\% \\
\hline 1 & $1: 2$ & 100 & 43 \\
2 & $1: 3$ & 100 & 70 \\
3 & $1: 6$ & 100 & 95 \\
4 & $1: 20$ & 100 & 40 \\
\hline
\end{tabular}

${ }^{a} \mathrm{H}_{2} \mathrm{O} 100 \mathrm{~mL}, \mathrm{H}_{3} \mathrm{PW}_{12} \mathrm{O}_{40} 0.1 \mathrm{~mol}, p$-nitrobenzaldehyde $0.15 \mathrm{~g}(1 \mathrm{mmol}), T=$ $25^{\circ} \mathrm{C}, t=48 \mathrm{~h}$.

分析表 1 的数据, 我们可以发现产物的产率在两者 比例过高或过低时均不高. 这可能是因为过多的吡咯自 身很容易发生齐聚反应生成聚吡咯而影响它与醛的缩 合, 过少则不能与醛完全反应, 而当它们的物质的量比 为 $1: 6$ 时, 产物的产率最高, 达到 $95 \%$. 粗产物的 ${ }^{1} \mathrm{H}$ NMR 分析结果表明, 我们得到的 5-(4-硝基苯基)二吡咯 甲烷的纯度大于 $99 \%$, 几乎没有副产物存在, 无需纯化 步骤.

为了排除反应物加入顺序因素的影响, 我们先改变 反应物和催化剂的添加顺序进行了一组对比实验 (Scheme 2), 反应进程用 TLC 跟踪监测.

TLC 分析结果表明, 底物醛在 $48 \mathrm{~h}$ 内完全转化, 得 到的产物的 $R_{\mathrm{f}}[V$ (石油醚) $: V$ (乙酸乙酯 $\left.)=5: 1\right]$ 值均为 0.2 , 熔点数据和 ${ }^{1} \mathrm{H}$ NMR 数据证明我们得到的单一产 物为 5-(4-硝基苯基)二吡咯甲烷. 这说明反应物的加入
顺序并没有对产物的种类和组成产生影响.

\section{2 催化剂用量}

为了深入研究催化剂用量对转化率和产率的影响, 我们在同样的实验条件下，使用不同量的催化剂进行了 重复实验(表 2).

表 $2 \mathrm{H}_{3} \mathrm{PW}_{12} \mathrm{O}_{40}$ 的用量对对硝基苯甲醛与吡咯缩合反应结果 的影响 ${ }^{a}$

Tabel 2 Effects of amount of $\mathrm{H}_{3} \mathrm{PW}_{12} \mathrm{O}_{40}$ on the pyrrole$p$-nitrobenzaldehyde condensation

\begin{tabular}{cccc}
\hline Amount of $\mathrm{H}_{3} \mathrm{PW}_{12} \mathrm{O}_{40} / \mathrm{mmol}$ & Time/h & Conversion $/ \%$ & Yield/\% \\
\hline 0.1 & 48 & 100 & 74 \\
0.5 & 48 & 100 & 95 \\
2 & 48 & 100 & 95 \\
5 & 48 & 100 & 86 \\
10 & 48 & 100 & 72 \\
\hline
\end{tabular}

${ }^{a} \mathrm{H}_{2} \mathrm{O} 100 \mathrm{~mL}, p$-nitrobenzaldehyde $1 \mathrm{mmol}$, pyrrole $6 \mathrm{mmol}, T=25{ }^{\circ} \mathrm{C}$.

分析表 2 的结果可以看出催化剂用量对转化率没有 明显影响, 但太低和太高的催化剂用量都会降低产物的 产率, 只有在适当的催化剂用量下才能达到最高的产 率.

这可能是因为, 在对硝基苯甲醛与吡咯反应的过程 中，首先是对硝基苯甲醛在适量 $\mathrm{H}^{+}$的诱导下形成了稳 定的羰基碳正离子，提高了对硝基苯甲醛的亲电性，大 大加强了它对吡咯 $\alpha$ 位的进攻能力, 生成亲核能力强的 亲电取代的中间体，这一步是该反应的控速步骤. 但过 高的 $\mathrm{H}^{+}$浓度会加剧吡咯的自身聚合反应生成聚吡咯而 降低产率, 过低的 $\mathrm{H}^{+}$浓度会使反应速度降低, 导致吡 咯自身聚合的几率增大.

\section{3 反应物浓度}

通常，较高的反应物浓度能增加反应分子间发生碰 撞的几率，提高产率; 但过高的反应物浓度同时也会促 进副反应的发生，阻碍目标产物的生成，降低产率.

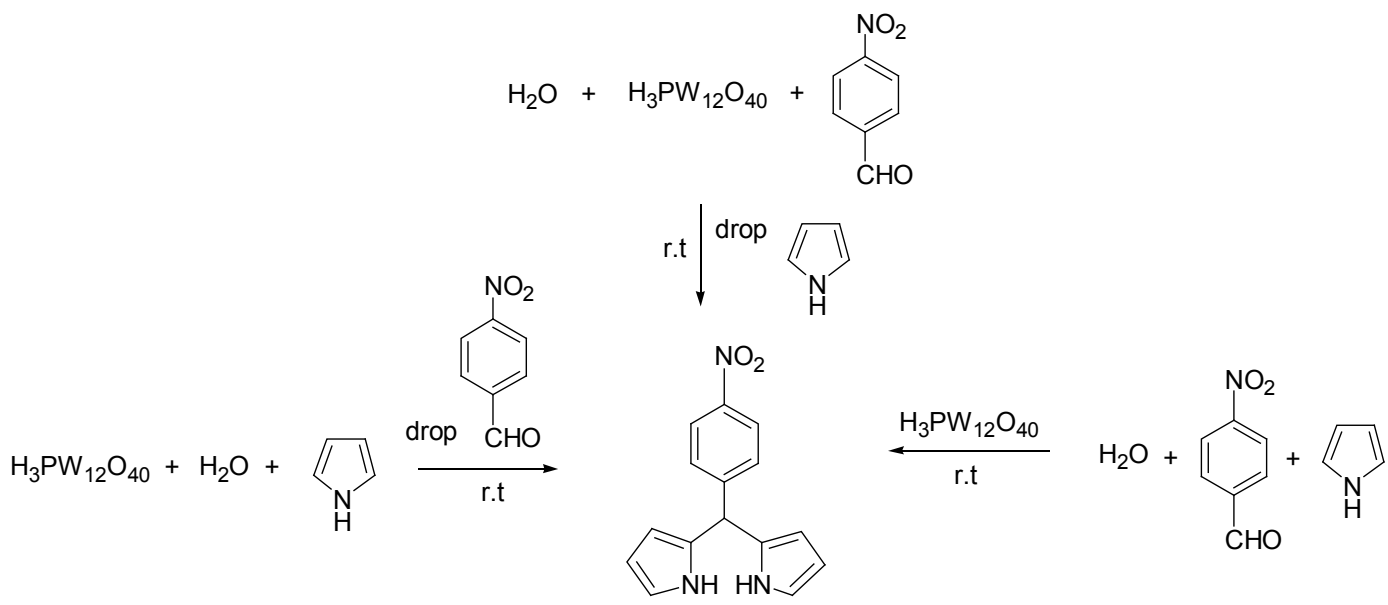

Scheme 2 
为了研究该反应中反应物浓度对反应转化率和产率的 影响，我们在其它条件不变的情况下，仅改变反应物的 浓度进行了实验, 结果如表 3 所示.

表 3 反应物浓度对对硝基苯甲醛与吡咯缩合反应结果的影 响

Tabel 3 Effectsof reactant concentration on the pyrrole$p$-nitrobenzaldehyde condensation

\begin{tabular}{cccc}
\hline Entry & $\begin{array}{c}p \text {-Nitrobenzaldehyde/ } \\
\left(\mathrm{mmol} \cdot \mathrm{L}^{-1}\right)\end{array}$ & Conversion/\% & Yield/\% \\
\hline 1 & 4.95 & 100 & 95 \\
2 & 9.8 & 100 & 95 \\
3 & 19.6 & 100 & 95 \\
4 & 39.2 & 100 & 95 \\
5 & 99 & 99 & 80 \\
6 & 247.5 & 85 & 52
\end{tabular}

${ }^{a} \mathrm{H}_{2} \mathrm{O} 100 \mathrm{~mL} ; \mathrm{H}_{3} \mathrm{PW}_{12} \mathrm{O}_{40} 0.1 \mathrm{mmol} ; T=25{ }^{\circ} \mathrm{C} ; t=48 \mathrm{~h}$.

表 3 的实验结果表明, 反应物的浓度有一个上限, 在最佳反应物比例不变的情况下, 过高的反应物浓度会 使吡咯发生自聚, 从而导致对硝基苯甲醛转化率和产率 急剧下降.

\section{4 醛或酮的种类}

室温下, 苯甲醛与吡咯在水中的溶解度较低, 二者 在水面上发生异相反应. 反应物 $\mathbf{1}$ 的反应活性为影响产 率和转化率的又一重要因素, 因此, 使用活性不同的 $\mathbf{1}$ 与吡咯在以上优化的反应条件下进行重复实验，得出的 结果如表 4 所示.

我们发现，采用正丙醛等脂肪醛在实验条件下得到 的是混合产物而不是单一产物，而当采用一些芳香醛及 酮类化合物进行反应时, $48 \mathrm{~h}$ 后得到了单一纯净的产物. 比较苯甲醛、对硝基苯甲醛和对羟基苯甲醛三种芳香醛 生成的产物的产率，我们发现活性较低的芳香醛的反应 产物产率较高; 比较丙酮、苯乙酮及对硝基苯乙酮、对 甲氧基苯乙酮、对氨基苯乙酮的生成产物的产率，我们 发现苯乙酮生成的产物的产率远大于取代苯乙酮，而取 代苯乙酮及丙酮生成产物的产率相近, 没有太大变化.

上述分析结果说明，活性较低的芳香醛及酮类化合 物在我们的实验条件下倾向于生成单一的二吡咯甲烷 类产物，而脂肪醛倾向于得到混合产物.

表 4 不同羰基化合物种类与吡咯缩合反应的结果 ${ }^{a}$

Tabel 4 The results of the reactions of pyrrole 2 with carbonyl compounds

\begin{tabular}{|c|c|c|c|}
\hline Compd. & Structure & Conversion/\% & Yield $^{b} / \%$ \\
\hline $1 \mathrm{a}$ & & 100 & 95 \\
\hline $1 b$ & & 100 & 55 \\
\hline $1 \mathrm{c}$ & & 100 & 53 \\
\hline 1d & & 100 & 62 \\
\hline $1 \mathrm{e}$ & & 100 & 97 \\
\hline 1f & & 100 & 59 \\
\hline $1 \mathrm{~g}$ & & 100 & 56 \\
\hline 1h & & 100 & 54 \\
\hline
\end{tabular}

${ }^{a} \mathrm{H}_{2} \mathrm{O}=100 \mathrm{~mL} ; \mathbf{1}: \mathbf{2}=1: 6 ; \mathrm{H}_{3} \mathrm{PW}_{12} \mathrm{O}_{40} 0.1 \mathrm{mmol} ; T=25{ }^{\circ} \mathrm{C} ; t=48 \mathrm{~h} ;{ }^{b}$ Isolated yield after purification.

\section{References}

[1] Pandey, R. K.; Jagerovic, N.; Ryan, M. J.; Dougherty, T. J.; Smith, K. M. Tetrahedron 1996, 52, 5349.

[2] Jasat, A.; Dolphin, D. Chem. Rev. 1997, 97, 2267.
[3] Gale, P. A.; Anzenbacher, P., Jr.; Sessler, J. L. Coord. Chem. Rev. 2001, 222, 57.

[4] Sessler, J. L.; Camiolo, S.; Gale, P. A. Coord. Chem. Rev. 2003, $240,17$.

[5] Wu, Y.-D.; Wang, D.-F.; Sessler, J. L. J. Org. Chem. 2001, 66, 3739 . 
[6] Wagner, R. W.; Johnson, T. E.; Lindsey, J. S. J. Am. Chem. Soc. 1996, $118,11166$.

[7] Lee, C.-H.; Lindsey, J. S. Tetrahedron 1994, 50, 11427.

[8] Laha, J. K.; Dhanalekshmi, S.; Taniguchi, M.; Ambroise, A.; Lindsey, J. S. Org. Process Res. Dev. 2003, 7, 799.

[9] Wijesekera, T. P. Can. J. Chem. 1996, 74, 1868.

[10] Nishino, N.; Wagner, R. W.; Lindsey, J. S. J. Org. Chem. 1996, 61, 7534.

[11] Littler, B. J.; Miller, M. A.; Hung, C.-H.; Wagner, R. W.; O'Shea, D. F.; Boyle, P. D.; Lindsey, J. S. J. Org. Chem. 1999, 64, 1391.

[12] Sobra, A. J. F. N.; Rebanda, N. G. C. L.; da Silva, M.; Lampreia, S. H.; Silva, M. R.; Beja, A. M.; Paixão, J. A.; Gonsalves, A. M. R. Tetrahedron Lett. 2003, 44, 3971.

[13] Král, V; Vašek, P; Dolenský, B. Collect. Czech. Chem. Commun. 2004, 69, 1126.

[14] Singh, K.; Behal, S.; Hundal, M. S. Tetrahedron 2005, 61, 6614.

[15] Temelli, B.; Unaleroglu, C. Tetrahedron 2006, 62, 10130.
[16] Rohand, T.; Dolusic, E.; Ngo, T. H.; Maes, W.; Dehaen, W. ARKIVOC (Gainesville, FL, United States) 2007, (10), 307.

[17] Pierre-Antoine Faugeras; Benjamin Boëns; Pierre-Henri Elchinger; Julien Vergnaud; Karine Teste; Rachida Zerrouki. Tetrahedron Lett. 2010, 51, 14630.

[18] Neya, S.; Funasaki, N. J. Heterocycl. Chem. 1997, 34, 689.

[19] Da Silva, F. M.; Jones, Jr J. J. Braz. Chem. Soc. 2001, 12, 135.

[20] Da, Silva F. M.; Gomes, A. K.; Jones, Jr. J. Can. J. Chem. 1999, 77, 624.

[21] Tsukinoki, T.; Tsuzuki, H. Green Chem. 2001, 3, 37.

[22] Tsukinoki, T.; Kanda, T.; Liu, G.-B.; Tsuzuki, H.; Tashiro, M. Tetrahedron Lett. 2000, 41, 5865.

[23] Liu, G.-B.; Tsukinoki, T.; Kanda, T.; Mitoma, Y.; Tashiro, M. Tetrahedron Lett. 1998, 39, 5991.

[24] Breton, G. W.; Hughey, C. A. J. Chem. Educ. 1998, 75, 85.

[25] Qiu, R. H.; Yin, S. F.; Zhang, X. W.; Xia, J.; Xu, X. H.; Luo, S. L. Chem. Commun. 2009, 4759. 